\title{
Uso de tecnologías de la informática y comunicación (TIC) para disminuir la deserción de egresados en posgrados semipresenciales
}

\author{
Víctor Hugo Méndez-Estrada y Zaidett Barrientos Llosa \\ Universidad Estatal a Distancia, 474 - 2050 San Pedro Montes de Oca, San José, Costa Rica; vmendez@uned.ac.cr; zbarrientos@uned.ac.cr
}

Recibido 9-II-2012 Corregido 23-V-2012 Aceptado 12-VII-2012

\begin{abstract}
Computer and communication technologies (ICT) help to reduce attrition in distance graduate education programs. Higher education dropout is a priority problem for university accreditation. It is also a frustration source for students and economic waste. The objective of this study was to determine whether the use of Information and Communication Technologies, in its broadest sense, can: reduce student dropout in the Master Program of Natural Resources Management at the Open University of Costa Rica, UNED, and increases its quality and linkage with society. We performed a comparative analysis of indicators before and after establishing two Information and Communication Technologies' Innovations: 1) creation of a research laboratory and 2) generation student research project progress monitoring databases. Analyzed periods are: a) 2002 to late 2007 and b) 2002 to late 2011. Analyzed indicators are related to students and professors academic activities and society impact (theses, informative and scientific publications amount). We found that in the first period $61 \%$ of graduates were inactive, while in the second period inactivity decreased to $17 \%$. Professors conducting research changed from zero to $36 \%$. Society impact increased from three to 18 theses, and from zero to 44 informative publications and 18 scientific papers. We conclude that the two Information and Communication Technologies' innovations applied, do support teaching, since attrition decreased and bonding with society increased.
\end{abstract}

\section{KEY WORDS}

graduate program, attrition, research, TIC'S.

\section{RESUMEN}

La deserción estudiantil es un problema que los programas de educación superior deben atender con prioridad, dado que provocan inconvenientes a la hora de la acreditación universitaria, son fuente de frustración para los estudiantes y constituyen una pérdida de recursos económicos. El objetivo de este estudio fue determinar si el uso de tecnologías de la informática y comunicación, en su sentido más amplio, permite disminuir la deserción estudiantil en la maestría de Manejo de Recursos Naturales de la Universidad Estatal a Distancia UNED (Costa Rica) y aumenta su calidad y vinculación con las necesidades de la sociedad. Realizamos un análisis comparativo de indicadores de dicha maestría antes del establecimiento de dos medidas y después de ellas. Las medidas de la informática y la comunicación que se aplicaron son: 1) creación de un laboratorio de investigación y 2) generación de bases de datos de seguimiento del avance de los proyectos de investigación de estudiantes. Los periodos analizados van del 2002 hasta finales del 2007 y los acumulados del 2002 hasta finales del 2011. Los indicadores analizados se relacionan con la actividad académica de estudiantes y docentes, y con la proyección hacia la sociedad (medida por las tesis y publicaciones científicas producidas durante esos periodos). Se encontró que en el primer periodo el $61 \%$ de los egresados estaban inactivos; mientras que el segundo la inactividad descendió a $17 \%$. La investigación realizada por los profesores pasó de cero a $36 \%$. La proyección a la sociedad pasó de solo tres a 18 tesis, y de cero a 44 publicaciones divulgativas y 18 artículos científicos. Se concluye que las dos medidas de uso de tecnología de la informática y comunicación si apoyan los procesos de enseñanza, ya que disminuye la deserción de egresados y favorece la vinculación del programa con la sociedad.

PALABRAS CLAVE

investigación, deserción, posgrados, TIC 
Uno de los problemas que los programas de educación superior deben atender con prioridad es el de la deserción de estudiantes (Díaz 2008). La deserción, además de ser un factor negativo en los procesos de acreditación universitaria (García 1987, Latiesa 1992, Dubs 2005), es considerada una fuente de frustración para los estudiantes (Dubs 2005) y una pérdida de recursos económicos para ellos y las universidades (Himmel 2002, Dubs 2005, Rivera et al. 2005). Los análisis sobre deserción se han llevado a cabo principalmente en programas de pregrado y de grado (Chyung et al. 1998, Lovitts \& Nelson 2000, Lovitts 2001, Himmel 2002). No obstante, los modelos teóricos desarrollados para el análisis de la deserción pueden ser aplicados también a posgrados universitarios (Barrientos \& Umaña 2009).

Por otra parte, en las entidades de educación superior cuando se habla sobre las tecnologías de información y comunicación (TIC) generalmente se analizan aspectos relacionados con el uso de computadoras: programas de cómputo, internet, plataformas virtuales, bases de datos, etc. (Monge-Nájera et al. 1999, Laborí \& Oleagordia 2001, Morales 2006, Borges 2007, Milachay 2007, Berrocal 2009, Brenes 2009). Sin embargo, en realidad el concepto de TIC es más amplio, incluye otras herramientas tecnológicas y de comunicación como el uso de equipo técnico especializado (Vidal 2006, Cabrero 2007), la comunicación de resultados a nivel técnico y divulgativo (González 2010) y la reestructuración curricular de la entrega de la docencia (Wirsig 2002, Fernández et al. 2004). Entonces, el uso de TIC en su sentido más amplio puede funcionar como mediación pedagógica para enfrentar algunas de las situaciones que motivan la deserción estudiantil.

La Maestría Académica en Manejo de Recursos Naturales de la Universidad Estatal a Distancia (UNED), Costa Rica, es un programa semipresencial que al ser académica exige como requisito de graduación que los estudiantes realicen una investigación con rigurosidad científica. El diseño curricular de la maestría incluye tres cursos de investigación cuyo fin es que los estudiantes elaboren su proyecto con la rigurosidad de un programa de maestría académica. Después, en coordinación con su tribunal de tesis el estudiante cuenta con seis meses para hacer una defensa oral y pública de su proyecto ante un tribunal evaluador (UNED 2010). Una vez aprobado el proyecto, el estudiante tiene dos años para elaborar su tesis, lo cual incluye tomar datos, analizarlos y redactar el documento final (UNED 2010). Barrientos \& Umaña (2009) encontraron que la mayor parte de los estudiantes interrumpieron sus estudios una vez que concluyeron los cursos, es decir, que su proyecto de investigación no llega a ser aprobado.
Un estudio previo (Barrientos \& Umaña 2009) y un análisis interno de la maestría determinaron que la deserción estudiantil está relacionada con cinco aspectos básicos: (a) mejorar la vinculación de la maestría con la problemática ambiental nacional y mundial; (b) vincular los dos énfasis de la maestría entre si; (c) articular mejor la relación entre los tres elementos básicos de las universidades -docencia, investigación y extensión-; (d) mejorar la formación de los estudiantes en procesos investigativos y (e) mejorar la habilidad didáctica e investigativa de los docentes.

Para solventar esas cinco debilidades se establecieron dos medidas basadas en el uso de tecnologías de la informática y comunicación: (1) creación de un laboratorio de investigación y (2) generación de bases de datos para seguimiento de los proyectos de investigación de los estudiantes. Por lo tanto, el propósito de este estudio es determinar si el uso de esas medidas disminuye la deserción estudiantil en la maestría de Manejo de Recursos Naturales y vincula al programa con la sociedad.

\section{METODOLOGÍA}

Se realizó un análisis comparativo con tres grupos de indicadores de los archivos con que cuenta la coordinación de la Maestría Académica en Manejo de Recursos Naturales de la UNED (Costa Rica), para evaluar: (a) la actividad académica de los estudiantes, (b) la investigación desarrollada por los docentes y (c) la vinculación que tiene la maestría con la sociedad. Estos indicadores fueron analizados en dos periodos: 2002 hasta finales del 2007 y los acumulados del 2002 hasta finales del 2011. Con base en ese análisis se incorporan dos medidas para el fortalecimiento de la maestría: (1) creación de un laboratorio de investigación y (2) generación de bases de datos para el seguimiento de los proyectos de investigación de los estudiantes. Este seguimiento estará a cargo de la coordinación de la Maestría en Manejo de Recursos Naturales. Estas medidas fueron aplicadas a inicios del 2008. El segundo periodo es acumulativo debido a que incluye a los estudiantes que no terminaron el programa en el tiempo reglamentario (2007), pero que motivados por los cambios realizados se mantuvieron activos durante el segundo periodo.

En la base de datos de seguimiento de los proyectos de investigación de los estudiantes se incluyen direcciones y números de teléfono del estudiante y de cada uno de sus miembros de comité de tesis, fecha de ingreso, si hubo interrupciones de estudio se registra la fecha y los motivos, así como cualquier otro detalle que caracterice o individualice la actividad académica del estudiante. En esta base también se lleva un control detallado del avance del 
proyecto de tesis del estudiante, se registra: las fechas en que entrega un documento, la fecha en que se le devuelve con correcciones, detalle de las correcciones y fecha en que el estudiante debe mandar una versión corregida, referencias sobre problemas y dificultades que los estudiantes expresan como motivos de su atraso y fechas aproximadas en las que ellos esperan resolver su dificultad. Cuando el estudiante se atrasa en la fecha estipulada para que envíe un avance o corrección se le manda un recordatorio personalizado, haciendo uso de la información almacenada en la base de datos de forma que el estudiante se siente apreciado, valorizado como individuo y perteneciente a la universidad. Además, se disminuyeron al mínimo los mensajes masivos e impersonales orientados a dar seguimiento al avance de los proyectos de investigación.

\section{RESULTADOS}

Las medidas tomadas estimulan la permanencia de estudiantes en el programa de maestría aun cuando el periodo reglamentario se ha vencido; propician la investigación docente y se establece una mayor vinculación del programa con la sociedad (Cuadro 1).

El porcentaje de estudiantes graduados, que elaboran tesis y desarrollan su proyecto de investigación ha aumentado (Cuadro 1).

El porcentaje de estudiantes egresados inactivos disminuyó de 86 a 24\%. Sin embargo, si el análisis de deserción se hace en relación con lo que estipula el reglamento, el porcentaje de desertores disminuyó en menor proporción (Cuadro 1).

Los indicadores relacionados con la articulación de la investigación (cantidad de profesores involucrados en investigaciones, cantidad de proyectos de investigación y publicaciones en revistas científicas) y la entrega de resultados de investigación a la sociedad (publicaciones divulgativas y talleres) demuestran un avance significativo (Cuadro 1).

El tiempo promedio transcurrido entre el egreso y la aprobación del proyecto de investigación ha aumentado considerablemente, pero el tiempo promedio transcurrido entre la aprobación del proyecto y la defensa de tesis ha disminuido ligeramente (Cuadro 2).

\section{Vinculación del plan de estudios de la maestría con la problemática ambiental nacional y mundial y vinculación de los dos énfasis de la maestría entre sí}

La generación de cinco proyectos de investigación sobre temáticas de interés nacional, la publicación de 44 trabajos dirigidos a la sociedad y la realización de dos simposios en la que los estudiantes y profesores exponen sus resultados garantiza la vinculación de la maestría y el laboratorio de ecología urbana con la realidad nacional (Cuadro 1).

Además, los estudiantes realizan sus tesis con temas de investigación en salud, educación, vulnerabilidad social, ambiente, biodiversidad, contaminación y calentamiento global, temas que son de gran interés en el país y el mundo entero.

\section{Vinculación de los tres elementos básicos del quehacer universitario: docencia, investigación y extensión}

Los procesos de investigación en los que participan docentes y estudiantes han dado como resultado la publicación de más de 18 publicaciones técnicas, 18 tesis y 44 presentaciones públicas en televisión, radio, periódicos y conferencias (Cuadro 1). La participación de los profesores en los proyectos de investigación ha permitido que enseñen con base en su propia experiencia. Todo esto, sin duda, es una muestra de la vinculación entre docencia, investigación y extensión.

\section{Formación de los estudiantes en procesos investigativos}

El Laboratorio de Ecología Urbana trabaja con medios tecnológicos tradicionales y de avanzada: cámaras, microscopios, microscopios estereoscópicos, sistemas de posicionamiento global (GPS), micro-estación meteorológica, hidrotermómetros, cintas diamétricas, software para sistemas de información geográfica, entre otros. Estos medios son importantes pues permiten a los estudiantes familiarizarse con equipos y tecnología que se están utilizando en los lugares de trabajo que ofrece la sociedad; por lo tanto, los estudiantes se incorporan en el mercado laboral con más facilidad.

Además, de la participación de algunos estudiantes en las investigaciones del laboratorio, se ha modificado la estructura de los cursos en los que elaboran su proyecto de investigación. El tener a un solo profesor permite darle seguimiento y continuidad al desarrollo del proyecto de investigación (tesis). Además, la especialidad del profesor en metodología de la investigación favorece la elaboración de mejores diseños experimentales. La generación de una base de datos en la que se registra el avance de cada estudiante permite dar un seguimiento individualizado a la formulación y desarrollo de tesis. 


\section{Habilidad didáctica e investigativa de los docentes}

La participación del 36\% de los profesores en procesos de investigación (cuadro 1) se liga directamente con la adquisición de nuevas habilidades en el desarrollo de investigaciones que pueden transmitir a los estudiantes.

\section{Deserción estudiantil del programa}

La deserción de estudiantes egresados disminuyó con las medidas tomadas, sin embargo, la disminución es menor si se toman en cuenta los tiempos establecidos en el reglamento de la UNED (Cuadro 1).

$\mathrm{Al}$ analizar el tiempo transcurrido entre el término de los cursos y la aprobación del proyecto de investigación se ve que ha aumentado (Cuadro 2). Sin embargo, una vez que se les aprueba el proyecto, el tiempo que transcurre hasta la defensa de la tesis ha disminuido, acercándose considerablemente al lapso establecido en el reglamento (Cuadro 2 ).

\section{DISCUSIÓN}

En ningún programa de maestría académica los estudiantes pueden ser únicamente receptores de información (Wirsig 2002, Clemenza 2004); por medio del uso de las TIC se conviertan en actores dinámicos, pues a través de ellas modifican rápidamente sus formas de vida, de interacción y producción (Monge-Nájera et al. 1999, Labori \& Oleagordia 2001, Aguilera 2005, Berrocal 2009, Brenes 2009), se les apoya para la investigación (Marcellán 2009, Luchilo 2010) y se les vincula con la docencia y extensión (Moreno 2005). El uso de las TIC a través de las dos medidas adoptadas (creación de un laboratorio de investigación y generación de bases de datos para el seguimiento de los proyectos de investigación), estimulan la permanencia del estudiante en el programa de maestría, aún después de vencido el periodo reglamentario. Esto hace que disminuya el porcentaje de estudiantes egresados inactivos; además, motiva a los docentes a participar en procesos de

CUADRO 1

Indicadores del nivel académico de la Maestría Académica en Manejo de Recursos Naturales, 2002-2011

\begin{tabular}{|c|c|c|c|}
\hline \multirow[t]{2}{*}{ Grupo de indicador } & \multirow[t]{2}{*}{ Indicador } & $\begin{array}{c}\text { Del } 2002 \text { a } \\
\text { diciembre } 2007 \\
(\mathrm{~N}=72)\end{array}$ & $\begin{array}{c}\text { Del } 2002 \text { a } \\
\text { diciembre } 2011 \\
\text { ( } N=92 \text { egresados })\end{array}$ \\
\hline & & $\%$ & $\%$ \\
\hline \multirow{5}{*}{$\begin{array}{l}\text { Actividad Académica de } \\
\text { los estudiantes }\end{array}$} & Estudiantes graduados & 4 & 20 \\
\hline & $\begin{array}{l}\text { Estudiantes egresados con el proyecto de } \\
\text { investigación aprobado y elaborando su tesis }\end{array}$ & 4 & 24 \\
\hline & $\begin{array}{l}\text { Estudiantes egresados elaborando su proyecto } \\
\text { de investigación }\end{array}$ & 6 & 33 \\
\hline & $\begin{array}{l}\text { Estudiantes egresados inactivos o deserción } \\
\text { voluntaria }\end{array}$ & 86 & 24 \\
\hline & Deserción de egresados por reglamento & 74 & 69 \\
\hline \multirow{2}{*}{$\begin{array}{l}\text { Investigación de los } \\
\text { docentes }\end{array}$} & Profesores involucrados en investigación $(n=14)$ & 0 & 36 \\
\hline & Proyectos de investigación elaborados & 0 & 5 \\
\hline \multirow{3}{*}{$\begin{array}{l}\text { Vinculación con la } \\
\text { sociedad }\end{array}$} & $\begin{array}{l}\text { Publicaciones en revistas científicas de } \\
\text { estudiantes y profesores }\end{array}$ & 0 & 5 \\
\hline & $\begin{array}{l}\text { Publicaciones divulgativas de los resultados de } \\
\text { investigación de profesores y estudiantes }\end{array}$ & 0 & 18 \\
\hline & $\begin{array}{l}\text { Organización de simposios y talleres en las } \\
\text { que exponen resultados de proyectos de } \\
\text { investigación tanto profesores como estudiantes }\end{array}$ & 0 & 44 \\
\hline
\end{tabular}




\section{CUADRO 2}

Tiempo promedio transcurrido entre el egreso, la aprobación del proyecto de investigación y la defensa de tesis.

\begin{tabular}{lcc}
\hline \multicolumn{1}{c}{ Indicador } & $\begin{array}{c}\text { Del 2002 a } \\
\text { diciembre 2007* }\end{array}$ & $\begin{array}{c}\text { Del 2002 a } \\
\text { diciembre 2011* }\end{array}$ \\
\hline $\begin{array}{l}\text { Tiempo promedio transcurrido entre el egreso y la aprobación } \\
\text { del proyecto de investigación }\end{array}$ & 0,28 años $(\mathrm{n}=3)$ & 1,7 años $(\mathrm{n}=40)$ \\
$\begin{array}{l}\text { Tiempo promedio transcurrido entre la aprobación del proyecto } \\
\text { de investigación y la defensa de tesis }\end{array}$ & 2,9 años $(\mathrm{n}=3)$ & 2,1 años $(\mathrm{n}=18)$ \\
* Promedio de tiempo transcurrido en años ( $\mathrm{n}=$ tamaño de muestra) & &
\end{tabular}

investigación y se logra una mayor vinculación del programa con la sociedad (McDonnell \& Picket 1990, Clemenza et al. 2004).

En nuestro caso, a pesar de que es en los ambientes urbanos donde se concentra la población humana, estos no han sido estudiados como un ecosistema (McDonnell \& Picket 1990). En los trópicos, en particular, es en donde menos investigaciones se han realizado $y$, por lo tanto, se desconocen los mecanismos ecológico-sociales que moldean las interacciones entre sociedad y ecosistemas urbanos. Las investigaciones universitarias (Padrón 2002, Rama 2007) como las que se realizan en el Laboratorio de Ecología Urbana estudian esas interrelaciones; por lo tanto, los dos énfasis de la maestría se ven integrados en estudios que contemplan tanto los aspectos biológicos como los ambientales y sociales de gran importancia para los costarricenses y el mundo. El laboratorio de investigación de Ecología Urbana logró integrar los dos énfasis de la maestría (Biodiversidad y Gestión Ambiental), al convertirse en un ente integrador de múltiples disciplinas: ambiente, salud, biodiversidad; patrones culturales y sociales; derecho ambiental, economía ambiental, geología, hidrología y educación ambiental, entre otras (Noriega \& Lechuga s.f.). Los estudiantes son guiados en sus proyectos de investigación por docentes, internos y externos de la UNED, conocedores de esos campos (Noriega \& Lechuga s.f., Castro 2004, Harrison et al. 2005, Guerrero 2007, Maldonado et al. 2007, León et al. 2009).

Los estudiantes graduados de esta maestría y los que están en proceso de elaborar la tesis realizan docencia, extensión e investigación por medio de su tesis (Padrón 2002, Rama 2007, Gayol et al. 2011). Ya que sus temas de investigación están vinculados con la problemática ambiental nacional y mundial, se logra así una mayor sensibilidad en lo profesional y, sus conocimientos propician una mejor gestión ambiental como lo propone la Conferencia Mundial de Educación Superior -CMES 2009- y la
Conferencia Regional de Educación Superior -CRES 2008(González 2010). Esto se logra principalmente porque sus temas de investigación (tesis) están orientados a resolver problemas de salud, educación, vulnerabilidad social, ambiente y calentamiento global; temas que son de interés para los países miembros de esas dos conferencias (González 2010).

La investigación en esas temáticas genera conocimiento nuevo (Castro 2004, León et al. 2009), prolonga la educación para la vida y es de interés para la sociedad, y a través de las TIC empleadas en este programa de maestría se logra motivar y satisfacer las expectativas educativas de estos estudiantes (Mayorga 1999, Ramírez 2000, Labori \& Oleagordia 2001, Cardona 2002, Méndez \& Nájera 2006, Berrocal 2009, Brenes 2009). Por medio del Laboratorio de Ecología Urbana se utiliza tecnología de avanzada que los estudiantes deben aprender a utilizar para poder integrarse en el mercado laboral que ofrece la sociedad. El uso de un laboratorio de investigación como herramienta pedagógica les da esa oportunidad, pues llegan a conocer el manejo de otras TIC como: sistemas de posicionamiento global (GPS), micro-estaciones meteorológicas, hidrotermómetros, cintas diamétricas y software para sistemas de información geográfica, entre otros.

Así, cuando estos estudiantes terminan el plan de estudios han adquirido conocimientos actualizados (Dulzaides \& Molina 2004, Harrison et al. 2005). Pero con los proyectos de tesis y su participación en propuestas de investigación se espera ir más allá y ofrecerles las competencias básicas para que sean entes generadores de conocimiento actualizado y que sean capaces de identificar, transformar e innovar procesos y procedimientos (Guerrero 2007) relacionados con la problemática ambiental de su sociedad o entorno cotidiano. Además, aprenden a usar herramientas de la información y comunicación para crear, adaptar, apropiar, manejar y transferir tecnologías a su campo de trabajo (Guerrero 2007), como lo requiere la 
sociedad (León et al. 2009, UNESCO 2009). Esas competencias, en primera instancia, solo se alcanzan con la elaboración y desarrollo de un buen proyecto de investigación o tesis (Gutiérrez \& Barrón 2008). Por lo tanto, las medidas tomadas para esta maestría específica demostraron que promueven efectivamente que los estudiantes continúen con la elaboración de su proyecto de investigación e inicien el desarrollo del mismo. Sin embargo, aún se tiene poco avance registrado entre el tiempo promedio transcurrido entre la aprobación del proyecto de investigación y la defensa de la tesis, debido a que los estudiantes una vez que completan su plan de estudios se reincorporan a su vida cotidiana y dedican poco tiempo a la elaboración de su tesis académica.

La incorporación de las dos medidas más la reorganización de los cursos de preparación de proyectos de tesis y la generación de bases de datos de seguimiento del avance de los proyectos de investigación de los estudiantes resultaron ser medidas eficaces para disminuir la deserción desde el punto de vista de la intención del estudiante. Con esas medidas, la inactividad o deserción voluntaria de estudiantes egresados se redujo al $24 \%$ y es probable que manteniendo las medidas y mejorándolas se pueda bajar la deserción por voluntad del estudiante al 19\% como lo proponen Barrientos \& Umaña (2009).

La divulgación de los conocimientos es una tarea primordial de todo investigador y estudiante de posgrado (Vence 2009), acción que se ha logrado con los estudiantes y docentes investigadores de esta maestría y, como bien claro lo expresa González (2010: 43) “Una de las maneras de evaluar la acción de estos investigadores es a través de los productos derivados de la investigación, sean por ejemplo publicaciones científicas y patentes." Al lograr que tanto docentes como estudiantes publiquen y divulguen los resultados de sus investigaciones se puede superar el 2,6\% de publicaciones con que contaba América Latina en el 2006, superada en mucho por Europa 42,1\% y Norteamérica con 31,8\% (González 2010). La producción académica de Costa Rica es muy incipiente y en el 2006 no llegaba a las 400 publicaciones (González 2010), por eso es indispensable establecer medidas que propicien la publicación de los resultados de las tesis de los estudiantes.

Para promulgar la generación de conocimiento nuevo es necesario que los programas de maestría desarrollen estrategias para disminuir la deserción y para que los estudiantes y docentes desarrollen investigación de calidad que culmine tanto en la publicación de artículos científicos como con la divulgación de la información a la sociedad. La generación de una base de datos de seguimiento y la creación de un laboratorio de investigación demostraron ser efectivas para ese fin. Estas medidas permiten darles a los estudiantes de maestrías semipresenciales la continuidad, seguimiento e integración a un grupo de investigación que los motive a concluir sus proyectos de investigación. Además, se logra poner a disposición de la sociedad los conocimientos nuevos que esta demanda.

\section{AGRADECIMIENTOS}

A Katya Calderón, por el apoyo para la creación del laboratorio y por contribuir con la visión global de la interacción del laboratorio con la maestría. Se le agradece también a Julián Monge-Nájera por la revisión del texto y a Maribel Zúñiga por la ayuda técnica.

\section{REFERENCIAS}

Aguilera, M.P. 2005. La investigación universitaria: indispensable enlosnuevostiempos.Universitas2000.29:11-12.(Disponible en http://www2.scielo.org.ve/scielo.php?script=sci_ arttext\&pid=S1315-41192005000200001\&lng=es\&nrm $=\mathrm{is})$.

Barrientos, Z. \& R. Umaña. 2009. Deserción estudiantil en posgrados semipresenciales de la Universidad Estatal a Distancia (UNED), Costa Rica: ¿Deserción o retraso? Cuadernos de Investigación UNED 1: 141-150. (Disponible en http:// www.uned.ac.cr/investigacio/publicaciones/cuaderno1/ documents/Barrientos_Desercion.pdf).

Berrocal, V. 2009. Consideraciones para el uso de simulaciones en entornos virtuales como apoyo del aprendizaje de las estrategias de programación de computadoras. EUNED, San José, Costa Rica. Innovaciones Educativas, año XI: 1-14.

Borges, F. 2007. El estudiante de entornos virtuales. Una primera aproximación. UOC Digithum 9. (Disponible en http:// www.uoc.edu/digithum/9/dt/esp/borges.pdf).

Brenes, O.L. 2009. Potencialidades de Internet como herramienta pedagógica en la educación superior. EUNED, San José, Costa Rica. Innovaciones Educativas, año XI:15-25.

Cabrero, J. 2007. Las necesidades de las TIC en el ámbito educativo: oportunidades, riesgos y necesidades. España, Tecnología y Comunicación Educativas 21. (Disponible en http://investigacion.ilce.edu.mx/tyce/45/articulo1.pdf).

Cardona, G. 2002. Tendencias educativas para el siglo XXI educación virtual, online y @learning elementos para la discusión. Edutec. Revista Electrónica de Tecnología Educativa 15. (Disponible en http://edutec.rediris.es/Revelec2/ Revelec15/car.htm).

Castro, G. 2004. Investigación, posgrado, y gestión del conocimiento. Algunos problemas y desafíos en el caso de Panamá. I Conferencia de Investigación y Posgrado, Universidad de Panamá. (Disponible en http://www.enlaceacademico. org/uploads/media/investigacion_posgrado_y_gestion_del_cononocimientos.pdf). 
Chyung, Y., D.Winiecki \&A. Fenner. 1998.Acasestudy:IncreaseenroIlment by reducing dropout rates in adult distanceeducation. (Disponible en http://www.eric.ed.gov/ERICDocs/data/ ericdocs-2sql/content_storage_01/0000019b/80/15/bf/ a9.pdf).

Clemenza, C., J. Ferrer \& R. Araujo. 2004. La investigación universitaria como vía de fortalecimiento de la relación universidad-sector productivo. Caso: Universidad del Zulia, Venezuela. Multiciencias 4. (Disponible en http://redalyc. uaemex.mx/src/inicio/ArtPdfRed.jsp?iCve=90440205).

Díaz, C. 2008. Modelo conceptual para la deserción estudiantil universitaria chilena. Estudios Pedagógicos 34:65-86. (Disponible en http://www.scielo.cl/scielo.php?pid=S071807052008000200004\&script=sci_arttext).

Dubs, R. 2005. Permanecer o desertar en los estudios de postgrado: Síntesis de modelos teóricos. Investigación y Postgrado 20: 55-79. (Disponible en: http://redalyc.uaemex.mx/src/inicio/ArtPdfRed.jsp?iCve $=65820103 \&$ iCveN $\mathrm{um}=3533$ )

Dulzaides, Ma. E. \& A. Ma. Molina. 2004. Análisis documental y de información: dos componentes de un mismo proceso. Centro de Información de Ciencias Médicas, Facultad de Ciencias Médicas de Cienfuegos, Cuba. (Disponible en http://bvs.sld.cu/revistas/aci/vol12_2_04/aci11204.htm).

Fernández, M.D., J. Rodríguez \& M.P. Vidal. 2004. La Influencia de las TIC en el desarrollo organizativo y profesional de un centro de primaria. Universidad Santiago de Compustela (USC), España. (Disponible en http://www.Imi.ub.es/edutec2004/pdf/182.pdf).

García, L. 1987. Rendimiento académico y abandono en la educación superior a distancia. Estudios de Educación a Distancia No. 10. Universidad Nacional de Educación a Distancia, Madrid, España.

Gayol, M.C., M.C. Tarrés, E. García \& A. Enrique. 2011. Aproximación sistemático-diacrónica para el desarrollo progresivo de competencias investigativas del saber-hacer en el grado y el postgrado del área salud. Revista Iberoamericana de Educación. (Disponible en http://www.rieoei.org/3712.htm).

González, E. 2010. El estado del arte de los posgrados e investigación latinoamericana y caribeña. Importancia de la CRES 2008, la CMES 2009 y el ENLACES. En Luchilo, L. (ed). Formación de posgrado en América Latina: políticas de apoyo. Resultados e impactos. Universitaria de Buenos Aires, Buenos Aires, Argentina, (Disponible en http://www.observatoriocts.org/files/Archivo\%20Documental/Libros\%20 del\%200bservatorio/formacion_de_postgrado.PDF).

Guerrero, Ma. E. 2007. Formación de habilidades para la investigación desde el pregrado. Acta Colombiana de Psicología 10: 190-192. (Disponible en http://regweb.ucatolica.edu. co/publicaciones/psicologia/ACTA/v10n2/articulosrevista/informacion\%20institucional.pdf).

Gutiérrez, N. G. \& Ma. C. Barrón. 2008. Tesis de posgrado en educación en el estado de Morelos. Temas y ámbitos de estudio. Universidad Nacional Autónoma de México, México, D.F. Perfiles Educativos XXX: 78-93. (Disponible en http:// redalyc.uaemex.mx/pdf/132/13211181004.pdf).
Harrison, L., A. Ray, R. Cianelli, M.S. Rivera \& M. Urrutia. 2005. Competencias en investigación para diferentes niveles de formación de enfermeras: una perspectiva latinoamericana. Revista Ciencia y Enfermería 11: 59-71. (Disponible en http://www.scielo.cl/scielo.php?pid=S071795532005000100007\&script=sci_arttext).

Himmel, E. 2002. Modelos de análisis para la deserción estudiantil en la educación superior. Calidad de la Educación: Retención y movilidad estudiantil en la educación superior. $2^{\circ}$ semestre. 91-107. (Disponible en http://www.cse.cl/ public/Secciones/seccionpublicacio-nes/doc/35/cse_articulo141.pdf).

Laborí, B. \& I. Oleagordia. 2001. Estrategias educativas para el uso de las nuevas tecnologías de la información y comunicación. OEI - Revista Iberoamericana de Educación. (Disponible en http://www.rieoei.org/deloslectores/Labori.PDF).

Latiesa, M. 1992. La deserción universitaria. Desarrollo de la escolaridad en la enseñanza superior. Éxitos y fracasos. Siglo XXI, España. (Disponible en http://www.cis.es/cis/opencm/ ES/3_publicaciones/catalogo/ver.jsp?id=64).

León, J., S. Sandoval \& S. López. 2009. Vinculación y transferencia de conocimiento de los investigadores de Sonora: un enfoque basado en la importancia de los factores individuales. Región y sociedad 21, México. (Disponible en http://www.scielo.org.mx/scielo. php?pid=S1870-39252009000200003\&script=sci_arttext).

Lovitts, B. \& C. Nelson. 2000. The Hidden Crisis in Graduate Education: Attrition from Ph.D. Programs, Academe 6: 44- 50. (Disponible en http://www.aaup.org/AAUP/pubsres/academe/2000/ND/Feat/lovi.htm?PF=1)

Lovitts, B. E. 2001. Leaving the Ivory Tower: The Causes and Consequences of Departure From Doctoral Study. Rowman \& Littlefield, Pensylvania, EEUU.

Luchilo, L. 2010. Formación de posgrado en América Latina: políticas de apoyo. Resultados e impactos. Universitaria de Buenos Aires, Buenos Aires, Argentina. (Disponible en http:// www.observatoriocts.org/files/Archivo\%20Documental/ Libros\%20del\%200bservatorio/formacion_de_postgrado.PDF).

Maldonado, L.F., D.P. Landazábal, J.C. Hernández, Y. Ruíz, A. Claro, H. Vanegas \& S. Cruz. 2007. Visibilidad y formación en investigación. Estrategias para el desarrollo de competencias investigativas. Revista Studiositas 2: 43- 56.

Marcellán, F. 2009. Investigación y universidad pública. Jornada de Reflexión y Debate, JUD2009, Madrid, España. (Disponible en http://www.jornadauniversidadpublica. org/?p=1097).

Mayorga, R. 1999. Los desafíos a la universidad latinoamericana en el siglo XXI. Organización de Estados Iberoamericanos (OEI). Revista Iberoamericana de Educación 21. (Disponible en http://www.rieoei.org/rie21a02.htm).

McDonnell, M.J. \& S.T.A. Picket. 1990. Ecosystem structure and function along urban-rural gradients: and unexploited opportunity for ecology. Ecology 71: 1232-1237. 
Méndez-Estrada, V. H. \& J. Monge-Nájera. 2006. Las TIC en un entorno latinoamericano de educación a distancia: la experiencia de la UNED de Costa Rica. RED. Revista de Educación a Distancia 15. Publicación en línea. Murcia, España. (Disponible en http://www.um.es/ead/red/15/).

Milachay, Y. 2007. Estrategias didácticas para el uso de las TIC en la enseñanza de postgrado. $I^{\mathrm{er}}$ Encuentro internacional de Educación Especial. Universidad Salazar, Instituto de estudios Superiores de Chiapas, México.

Monge-Nájera, J., M. Rivas Rossi \& V.H. Méndez-Estrada. 1999. Internet, multimedia and virtual laboratories in $a$ "third world" environment: how we solved the 21 basic problems in the Costa Rican distance education university. Universidad Estatal a Distancia, San José, Costa Rica.

Morales, V. 2006. La universidad latinoamericana actual: necesidad de replantear su misión. Caracas, Venezuela, Saber.ula.ve. Universidad Central de Venezuela, Centro de Estudios e Investigaciones sobre Educación Avanzada (CEISEA). Revista Mundo Universitario 7. (Disponible en http://www.saber.ula.ve/bitstream/123456789/22144/1/ articulo9.pdf).

Moreno, L. 2005. Modelo degerencia del conocimientoatravés dela extensiónuniversitaria.InvestigaciónyPostgrado20.Caracas, Venezuela. (Disponible en http://www.scielo.org.ve/scielo. php?pid=S1316-00872005000200008\&script=sci_arttext).

Noriega, M. \& S. Lechuga. s.f. Producción y circulación del conocimiento científico educativo en posgrados para profesores de educación básica: el Instituto Superior de Ciencias de la Educación del Estado de México. X Congreso Nacional de Investigación Educativa. (Disponible en http://www. comie.org.mx/congreso/memoria/v10/pdf/area_tematica_04/ponencias/0915-f.pdf).

Padrón, J. 2002. El problema de organizar la investigación universitaria. Universidad Peruana Cayetano Heredia, Diálogos Universitarios de Postgrado 11:9-33. (Disponible en http:// padron.entretemas.com/Organizar_IU.htm).

Rama, C. 2007. La educación superior en América Latina: posgrado e investigación. Escuela de Posgrado,
Universidad Nacional de Cajamarca, Cajamarca, Perú. (Disponible en http://www.slideshare.net/claudiorama/ los-postgrados-y-la-investigacin-universitaria)

Ramírez, P. 2000. Algunas consideraciones en torno a la misión y a las grandes áreas del quehacer universitario: el caso de la UNED. II Congreso Universitario por un Cambio con Participación Real, 2-11 noviembre, San José, Costa Rica

Rivera, E., H. Roca, B. Echart, A. López, S. Sossa, C. Mercado \& B. Barrera. 2005. Estudio sobre repitencia y deserción en la educación superior en Bolivia. UNESCO. (Disponible en http:// unesdoc.unesco.org/images/0013/001397/139799s.pdf).

UNED. 2010. Reglamento de graduación de las maestrías académicas del sistema de estudios de posgrado. UNED, San José, Costa Rica.(Disponible en http://www.uned.ac.cr/ Reglamento/documents/GRADUACIONMAESTRIASACADEMICASDELSISTEMADEESTUDIOSDEPOSGRADOAGOSTO05.pdf).

UNESCO. 2009. 2009 World Conference on Higher Education: The New Dynamics of Higher Education and Research For Societal Change and Development (UNESCO, Paris, 5 - 8 July 2009) (Disponible en http://www.unesco.org/fileadmin/ MULTIMEDIA/HQ/ED/ED/pdf/WCHE_2009/FINAL\%20 COMMUNIQUE\%20WCHE\%202009.pdf).

Vence, X. 2009. La investigación universitaria frente al corsé de las patentes, la mercantilización del conocimiento y la empresa privada. Jornada de Reflexión y Debate, JUD2009, Madrid, España. (Disponible en http://www.jornadauniversidadpublica.org/?p=1217).

Vidal, M.P. 2006. Investigación de las TIC en educación. Revista Latinoamericana de Tecnología Educativa. 5: 539-552. (Disponible en http://www.unex.es/didactica/RELATEC/ sumario-5-2.htm).

Wirsig, S. 2002. ¿Cuál es el lugar de la tecnología en la educación? (Disponible en http://es.scribd.com/doc/42345/ tic-en-educacion). 